\title{
FIB-SEM 3D Spectral Imaging for Materials Characterization
}

\author{
Paul G. Kotula \\ Sandia National Laboratories, PO Box 5800, Albuquerque, NM 87185-0886
}

Tomographic spectral imaging is a powerful technique for the 3D analysis of materials. The present work describes the application of this technique to the analysis of a braze joint between Ag-2Zr and Alumina-Glass ceramic. Implemented via serial sectioning in a focused ion-beam/scanning electron microscope, electron-excited x-ray spectra were acquired from each voxel in a 3D array [1]. The resultant tomographic spectral image was analyzed in its entirety with Sandia's Automated eXpert Spectral Image Analysis (AXSIA) multivariate statistical analysis software [1-3]. The result of the analysis is a small number of chemical components which describe the 3D phase distribution in the volume of material sampled.

An FEI Company DB-235, dual beam SEM/FIB equipped with a Thermo NORAN Si(Li) energy-dispersive $\mathrm{x}$-ray spectrometer (EDS) was utilized for this work. The FIB was operated at $30 \mathrm{kV}$ with $\mathrm{Ga}$ as the source for ions. The SEM was operated at $5 \mathrm{kV}$ to maximize the spatial resolution of the $\mathrm{x}$-ray signal. Given this relatively low accelerating voltage and the low spectral resolution of the EDS (150 eV at $\mathrm{MnK} \alpha$ ), numerous pathological overlaps are expected amongst the available x-ray lines. The solution to this problem comes from the use of advanced multivariate statistical analysis methods [1-3]. The primary benefit of working at low accelerating voltages is that spatial resolution is improved and therefore thinner serial section thicknesses (down to $250 \mathrm{~nm}$ or lower) are practical without the likelihood of information coming from deeply buried structures as would happen at $30 \mathrm{kV}$ or $15 \mathrm{kV}$ for example.

Multivariate statistical analysis implemented with Sandia's AXSIA software [1-3] was applied to the TSI data without foreknowledge of the presence or absence of specific elements or the specific shapes of the x-ray emission lines. Figure 1 shows five (out of eight) of the spectral shapes derived from the analysis of the TSI. Firstly the shapes of the spectra are all positive, unlike, for example, principal components analysis. Secondly, associations between elements are readily apparent, such as $\mathrm{Na}, \mathrm{Mg}$, and Ca present only in the glass with $\mathrm{Al}, \mathrm{Si}$, and $\mathrm{O}$, which are also found elsewhere. This analysis has also distinguished two different phases containing $\mathrm{Zr}$, zirconium oxide and zirconium silicide.

To appreciate the 3D relationships between the respective components, they were rendered with Imaris ${ }^{\mathrm{TM}}$ from Bitplane AG. No smoothing of the data was performed in the rendering process. Figure 2 shows a $3 \mathrm{D}$ rendering of the components. The volume of the material sampled in the analysis was $40 \mu \mathrm{m}$ by $16 \mu \mathrm{m}$ by $5 \mu \mathrm{m}$ respectively for a total volume sampled of $3200 \mu^{3}$. It is interesting to note that a void between the metal and ceramic was sampled and it is clear that where the metal did not wet the ceramic, zirconium silicide (magenta) was able to form. Where the metal wet the ceramic, 
zirconium oxide (yellow) instead formed. As a result of the processing, however, the zirconium oxide separates from the ceramic interface and thus the measurements here can give an accurate measure of amount of zirconium oxide in contact with the substrate which can then be related to the bonding strength of the braze joint.

[1] P.G. Kotula, M.R. Keenan and J.R. Michael. Microsc. Microanal. (2006) 12 36-48.

[2] P.G. Kotula, M.R. Keenan and J.R. Michael. Microsc. Microanal. (2003) 9 1-17.

[3] M.R. Keenan and P.G. Kotula, Microsc. Microanal. (2004) 10 [Suppl. 2 Proc] 874CD. The author would like to thank Michael Rye at Sandia for FIB serial sectioning and spectral image acquisition. Sandia is a multiprogram laboratory operated by Sandia Corporation, a Lockheed Martin Company, for the United Stated Department of Energy's (DOE) National Nuclear Security Administration (NNSA) under contract DEAC0494AL85000.

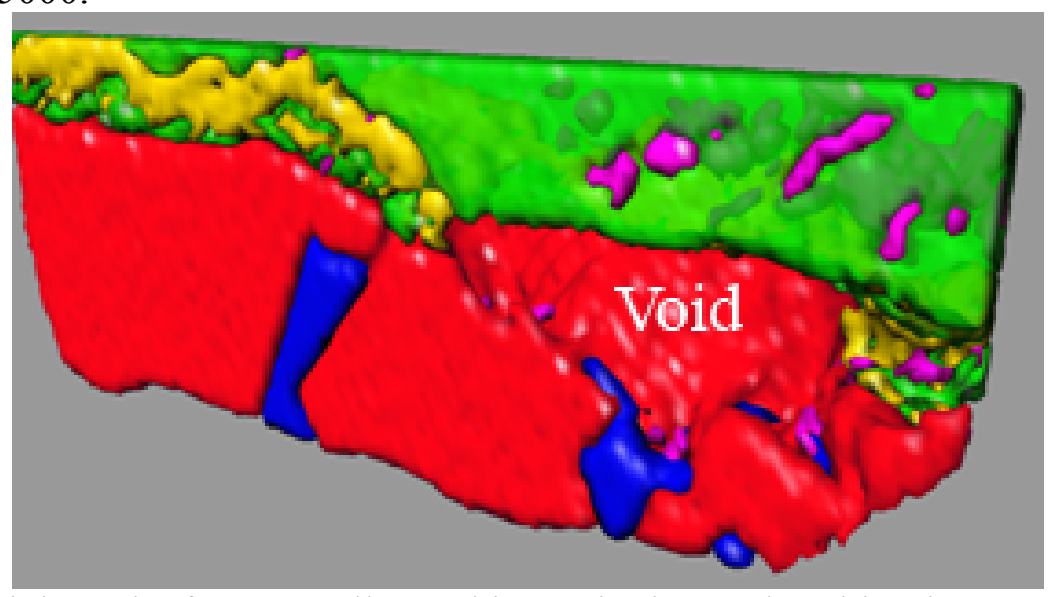

Figure 1. Partial results from metallographic sectioning and multivariate statistical analysis of the Ag-2Zr-to-Alumina/Glass Ceramic. $\mathrm{Red}=\mathrm{Al}_{2} \mathrm{O}_{3}$, green $=\mathrm{Ag}$, blue $=$ Glass, magenta $=\mathrm{Zr}-\mathrm{Si}$, and yellow $=\mathrm{Zr}-\mathrm{O}$.

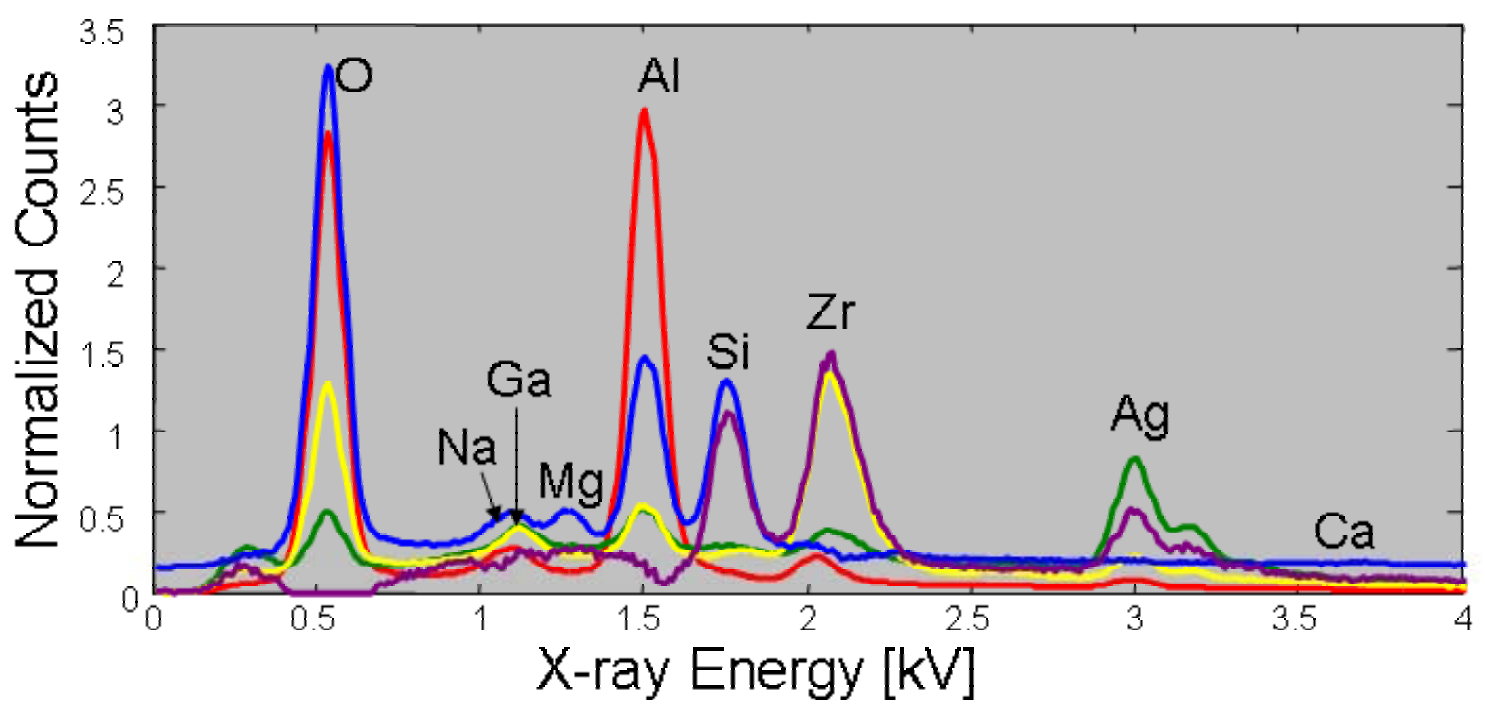

Figure 2. Spectral shapes for the components shown in Fig. 1. Note that several overlapping peaks and weak spectral features are clearly visible. 